\title{
Harrison-Zeldovich spectrum from conformal invariance
}

\author{
V.A. Rubakov \\ Institute for Nuclear Research of the Russian Academy of Sciences, \\ 60th October Anniversary Prospect, 7a, 117312 Moscow, Russia
}

\begin{abstract}
We show that flat spectrum of small perturbations of field(s) is generated in a simple way in a theory of multi-component scalar field provided this theory is conformally invariant, it has some global symmetry and the quartic potential is negative. We suggest a mechanism of converting these field perturbations into adiabatic scalar perturbations with flat spectrum.
\end{abstract}

\section{Introduction}

An elegant mechanism of the generation of (almost) flat spectrum of scalar perturbations is provided by inflation [1]. Yet it is legitimate to ask whether the spectrum of the HarrisonZeldovich type can emerge in a different way. This question is of interest, in particular, from the viewpoint of alternatives to inflation - pre-Big Bang scenario [2] (for a review see Ref. [3]), ekpyrotic/cycling models [4, 5] (for a review see Ref. [6]), starting-the-Universe picture [7], etc. One possibility is to make use of scalar fields with negative exponential potentials [8, 9, 10, 11] (see also Ref. [12]). In this way one indeed obtains almost flat spectrum of scalar perturbations in ekpyrotic models, with overall homogeneity and isotropy of the Universe preserved during the contracting phase because of the stiff effective equation of state [13]. In concrete models of this type the contracting solutions are unstable [14, 15], so that one has to fine tune initial data in our part of the Universe. It has been argued, however, that such a fine tuning may be automatic, in a sense, in a cyclic scenario with long periods of dark energy domination [16].

In this paper we suggest another mechanism for generating the Harrison-Zeldovich spectrum. The idea is to relate scale invariance of this spectrum to conformal invariance of field theory. We will see in Section 2 that this can be done by introducing conformal scalar field as a source of entropy perturbations, which are converted into adiabatic perturbations at 
later epoch. The three basic ingredients are that (i) the theory possesses some global symmetry, (ii) the quartic scalar potential is negative and (iii) there is long enough stage of the cosmological evolution prior to the conventional hot Big Bang epoch. At that early stage, the classical scalar field rolls down its potential along the late time attractor. In this regime, the vacuum perturbations of field(s) orthogonal to the radial direction - the direction along which the classical field rolls down - get amplified and freeze out having the spectrum which is automatically flat. Hence, getting flat spectrum of the field perturbations is reasonably simple.

Implementing this mechanism to obtain adiabatic matter perturbations of correct amplitude is somewhat less straightforward. We propose the corresponding scenario in Section 3. At the end of the rolling stage, both conformal invariance and global symmetry are assumed to be broken, and the scalar potential is assumed to have a minimum or a set of minima, see Fig. 1. If the scale of global symmetry breaking is small enough, the situation becomes analogous to the axion misalignment picture (for a review see Ref. [17]), the analog of axion being the orthogonal, pseudo-Goldstone field(s) corrseponding to broken global symmetry. Unlike in the case of axion, the interactions of the pseudo-Goldsone field with matter are not negligible, so this field plays the same role as curvaton in inflationary models. It gets frozen at a slope of the potential, later on it starts to oscillate and eventually these oscillations convert into conventional particles. In this way the adiabatic perturbations with flat spectrum are produced.

In Section 4 we discuss a subtle point of our model. Namely, at the stage when the radial field rolls down, this field develops its own perturbations. Their growth is fast, and at some point the linearized theory breaks down. We argue, however, that the results of the linearized theory concerning the perturbations of the pseudo-Goldstone field remain valid, and the flat spectrum of adiabatic perturbations is generated indeed.

We end up in Section 5 with concluding remarks.

\section{Getting flat spectrum}

To begin with, let us consider massless scalar field $\phi$ conformally coupled to gravity and evolving in spatially flat 4-dimensional FRW Universe. Let us assume for the time being that there is exact global symmetry, which we take for definiteneness to be $U(1)$ (though the argument is valid for any compact symmetry group). Hence, the field is comlex, and the global symmetry is $\phi \rightarrow \mathrm{e}^{i \alpha} \phi$. Conformal invariance allows for quartic scalar potential, which we assume to be negative,

$$
V(\phi)=-h^{2}|\phi|^{4}
$$




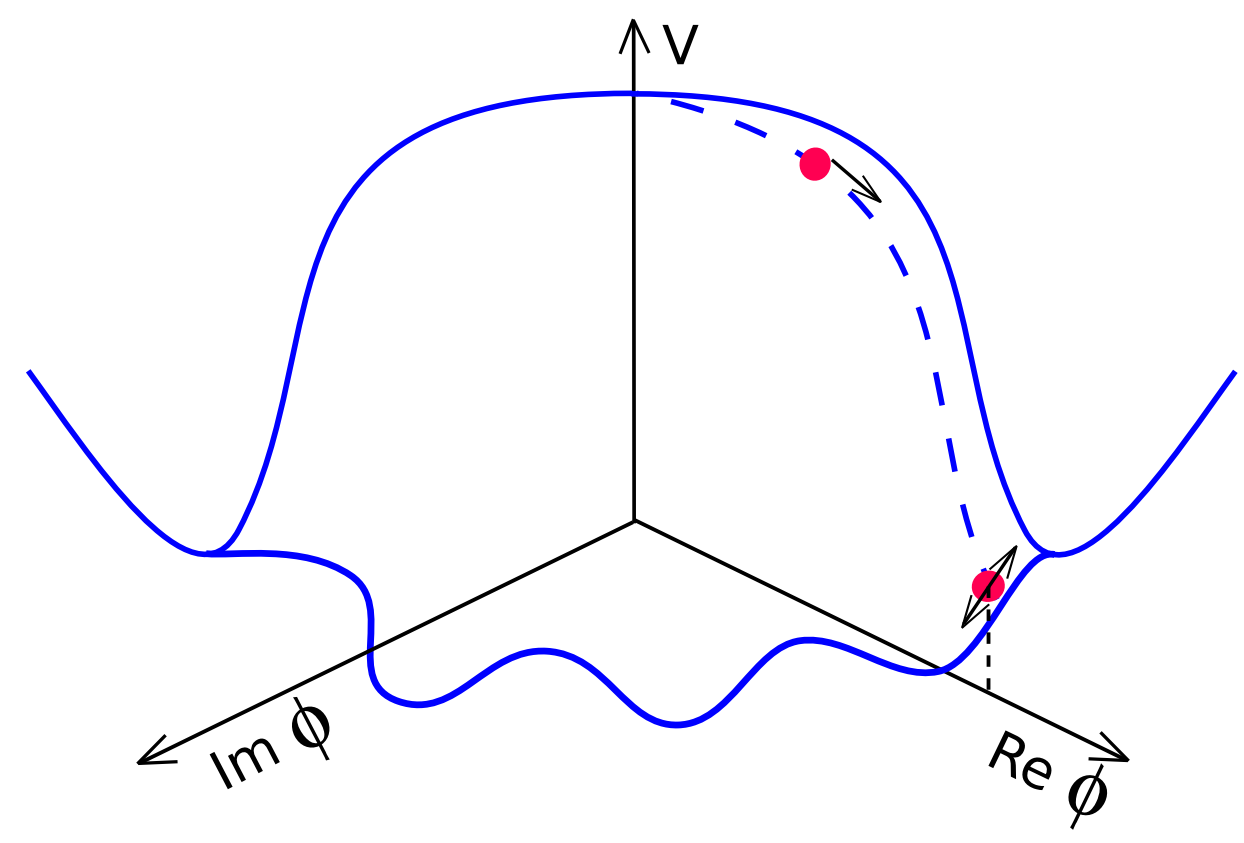

Figure 1: The scalar potential is negative quartic and respects global symmetry $(U(1)$ here) at relatively small $\phi$, and has one or more minima at large $\phi$. Dashed line shows the evolution of the field (dot with arrow) at the rolling stage. At that stage the perturbations (shown by double arrow) of the pseudo-Goldstone field are developed. At the hot Big Bang epoch, the pseudo-Goldstone field transfers its energy to hot matter, and its perturbations are converted into adiabatic perturbations.

(modulo an additive constant). As usual, one defines the field $\chi$ by

$$
\phi=\frac{\chi}{a} \equiv \frac{\chi_{1}+i \chi_{2}}{a}
$$

Then the action for $\chi$ in conformal coordinates is

$$
S_{\chi}=\int d^{3} x d \eta\left[\partial_{\mu} \chi^{*} \partial^{\mu} \chi+h^{2}|\chi|^{4}\right]
$$

where indices are raised by Minkowski metric.

Let us consider spatially homogeneous background field. The field equation is

$$
-\chi^{\prime \prime}+2 h^{2}|\chi|^{2} \chi=0
$$

where prime denotes $d / d \eta$. In terms of the radius and phase, $\chi=\rho \mathrm{e}^{i \theta}$, one of the equations is the conservation of current,

$$
\frac{d}{d \eta}\left(\rho^{2} \theta^{\prime}\right)=0
$$


Hence, as the value of $\rho$ increases, the phase $\theta$ freezes out, and the evolution proceeds along the radial direction. Without loss of generality we take $\chi$ to be real at that time, $\chi=\chi_{1}$. Then the first integral of the equation of motion is $\chi_{1}^{\prime 2}-h^{2} \chi_{1}^{4}=\epsilon=$ const. Hence, the solutions approach the asymptotics that is independent of $\epsilon$,

$$
\chi_{1}(\eta)=\frac{1}{h\left(\eta_{*}-\eta\right)},
$$

where $\eta_{*}$ is a constant of integration.

Now, let us study field perturbations in the orthogonal direction, $\delta \chi_{2}$. They obey the linearized equation, in momentum representation,

$$
\left(\delta \chi_{2}\right)^{\prime \prime}+k^{2} \delta \chi_{2}-2 h^{2} \chi_{1}^{2} \delta \chi_{2}=0 .
$$

At early times, when $k\left(\eta_{*}-\eta\right) \gg 1$, the second term dominates, and $\delta \chi_{2}$ oscillates like free scalar field in Minkowski space-time. At later times the third term dominates instead. So, the perturbation "exits the horizon", but now the "horizon" is due to evolving $\chi_{1}$ (hereafter we use quotation marks to distinguish this "horizon" from the true cosmological horizon). Outside the "horizon", i.e., at $k\left(\eta_{*}-\eta\right) \ll 1$, the perturbation $\delta \chi_{2}$ evolves in the same way as the background $\chi_{1}$. This is clear from the fact that at $k=0$, eq. (3) is the linearized equation (1). Now, alongside with the solution $\chi_{1}(\eta)$, eq. (1) has a solution $\mathrm{e}^{i \alpha} \chi_{1}(\eta)$, where $\alpha$ is a real constant. For small $\alpha$ the latter solution is $\left(\chi_{1}+i \alpha \chi_{1}\right)$, and the second part is small perturbation, which is precisely $\delta \chi_{2}$. So, if the perturbation $\delta \chi_{2}$ oscillates with amplitude $c(k)$ at early times, it behaves at late times as

$$
\delta \chi_{2}=C c(k) \chi_{1}(\eta)=C^{\prime} \frac{c(k)}{k\left(\eta_{*}-\eta\right)},
$$

where the factor $k^{-1}$ is evident on dimensional grounds, $C$ and $C^{\prime}$ are time-independent, and $C^{\prime}$ is independent of $k$. This is precisely the enhancement of perturbations needed for getting flat spectrum of $\delta \chi_{2}$. We stress that this property is entirely due to the symmetries of the model: the behaviour (2) of the background is a consequence of conformal invariance, whereas the behaviour (4) of perturbations is a consequence of the global $U(1)$ symmetry.

In more detail, eq. (3) reads

$$
\left(\delta \chi_{2}\right)^{\prime \prime}+k^{2} \delta \chi_{2}-\frac{2}{\left(\eta_{*}-\eta\right)^{2}} \delta \chi_{2}=0 .
$$

This is formally the same equation as the equation for perturbations of minimally coupled massless scalar field in de Sitter space-time. Hence, the spectrum of $\delta \chi_{2}$ is the same flat spectrum. We are interested in the solution that behaves at early times as

$$
\chi_{2}^{(-)}=\frac{1}{(2 \pi)^{3 / 2} \sqrt{2 k}} \mathrm{e}^{i k\left(\eta_{*}-\eta\right)} .
$$


Modulo overall constant phase, this solution is expressed through the Hankel function,

$$
\chi_{2}^{(-)}=\frac{1}{4 \pi} \sqrt{\frac{\eta_{*}-\eta}{2}} H_{3 / 2}^{(1)}\left[k\left(\eta_{*}-\eta\right)\right] .
$$

At $k\left(\eta_{*}-\eta\right) \ll 1$ this solution is

$$
\chi_{2}^{(-)}=\frac{i}{2 \pi^{3 / 2}} \frac{1}{k^{3 / 2}\left(\eta_{*}-\eta\right)},
$$

in accord with (4). Assuming that the quantum field $\delta \chi_{2}$ is originally in the vacuum state, we obtain its power spectrum outside the "horizon" in the standard way. The spectrum is flat, and its amplitude is

$$
\Delta_{\chi_{2}} \equiv \sqrt{\mathcal{P}_{\chi_{2}}}=\frac{1}{2 \pi\left(\eta_{*}-\eta\right)} .
$$

As promised, generating flat spectrum of the field perturbations is fairly straightforward.

We end up this section by noting that the mechanism requires long time during which the classical field $\phi$ rolls down its potential. Indeed, let $\eta_{i}$ be the time at which the field starts to roll down, and $\eta_{f}$ the time at which rolling down terminates. Then the existence of the regimes (5) and (6) requires that

$$
\begin{aligned}
& k\left(\eta_{*}-\eta_{i}\right) \gg 1, \\
& k\left(\eta_{*}-\eta_{f}\right) \ll 1 .
\end{aligned}
$$

These inequalities should hold for all scales of interest, say, for present momenta (we set the present value of the scale factor equal to 1 )

$$
k_{\min } \sim(1 \mathrm{Gpc})^{-1} \lesssim k \lesssim k_{\max } \sim(100 \mathrm{kpc})^{-1}
$$

Therefore, the duration of the rolling stage should be large,

$$
\left(\eta_{f}-\eta_{i}\right) \gg\left(k_{\min }^{-1}-k_{\max }^{-1}\right) \approx k_{\min }^{-1}
$$

This excludes the possibility that the mechanism works at the hot stage of the cosmological evolution: there is simply not enough time. On the other hand, there may well be enough time in the ekpyrotic or starting-the-Universe scenario ${ }^{1}$.

\footnotetext{
${ }^{1}$ Note that the inequality (9) means that the comoving size of light cone originating from $\eta=\eta_{i}$ and measured at $\eta=\eta_{f}$ exceeds the comoving size of the visible Universe. Hence, the mechanism can work only in cosmological models which solve, at least formally, the horizon problem.
} 


\section{Implementing the mechanism}

In this Section we suggest a way to implement the mechanism and generate adiabatic perturbations from the perturbations $\delta \phi_{2}$. Let us assume that as the classical field $\phi_{1}=\chi_{1} / a$ approaches the region $\phi_{1} \sim f$, conformal symmetry gets broken and the potential along the radial direction has a minimum at $\phi_{1}=f$, where $f$ is some high energy scale. We assume that $O(2)$ symmetry is broken as well, but that the scale of that breaking is small compared to $f$, so we neglect the effects of $O(2)$ symmetry breaking for the time being. Then the classical field $\phi_{1}$ oscillates near $f$ and eventually settles down to $\phi_{1}=f$ (say, by producing conventional particles). We require that this process occurs at the time when the energy density of the field $\phi_{1}$ is much smaller than the total energy density in the Universe. The reason for this requirement is that the field $\phi_{1}$ develops its own perturbations. In terms of perturbations $\delta \chi_{1}$, the linearized equation for this mode is

$$
\left(\delta \chi_{1}\right)^{\prime \prime}+k^{2} \delta \chi_{1}-\frac{6}{\left(\eta_{*}-\eta\right)^{2}} \delta \chi_{1}=0 .
$$

The solution which is negative frequency at early times is again expressed through the Hankel function, but with another index,

$$
\chi_{1}^{(-)}=\frac{1}{4 \pi} \sqrt{\frac{\eta_{*}-\eta}{2}} H_{5 / 2}^{(1)}\left[k\left(\eta_{*}-\eta\right)\right] .
$$

At $k\left(\eta_{*}-\eta\right) \ll 1$ this solution is

$$
\chi_{1}^{(-)}=-\frac{i}{\pi \Gamma(-3 / 2)} \frac{1}{k^{5 / 2}\left(\eta_{*}-\eta\right)^{2}} .
$$

This behaviour again has simple interpretation: if $\chi_{1}$ is a classical solution to the field equation (1), then

$$
\delta \chi_{1} \propto \chi_{1}^{\prime}
$$

is a solution to the linearized classical field equation for $k \rightarrow 0$. Hence $\delta \chi_{1} \propto\left(\eta_{*}-\eta\right)^{2}$ on super-"horizon" scales; the delpendence on $k$ again follows from dimensional argument. In any case, eq. (10) implies that the spectrum of perturbations of the field $\chi_{1}$ is red,

$$
\Delta_{\chi_{1}} \equiv \sqrt{\mathcal{P}_{\chi_{1}}}=\frac{3}{2 \pi k\left(\eta_{*}-\eta\right)^{2}}
$$

These perturbations should not leave any trace in the late Universe; the simplest way to achieve that is to impose the above requirement.

The perturbations of the field $\phi_{1}$ may become so strong that they ruin the linearized analysis. To be on the safe side, we impose here the requirement that the field $\phi_{1}$ is approximately homogeneous over the whole visible Universe at the time when the scales of interest 
exit the "horizon" (see, however, Section 4). This is sufficient for the linearized theory to be valid in our patch of the Universe at that time; perturbations $\delta \phi_{1}$ of longer wavelengths correspond to unobservable shift of the parameter $\eta_{*}$ in the whole visible Universe, see (11). Our requirement gives

$$
\Delta_{\chi_{1}}\left(k_{\min }, \eta_{\max }\right) \ll \chi_{1}\left(\eta_{\max }\right),
$$

where $k_{\min }^{-1}$ is the comoving size of the visible Universe, cf. (8), and $\eta_{\max }$ is the time of the "horizon" exit of the shortest interesting modes. The value of $\eta_{\max }$ is determined by

$$
k_{\max }\left(\eta_{*}-\eta_{\max }\right) \sim 1
$$

Making use of (2) and (12) we obtain a constraint on the coupling constant

$$
h \ll \frac{k_{\min }}{k_{\max }} \sim 10^{-4} .
$$

We will further discuss the non-linearity issue in Section 4.

Let us now turn to the field $\phi_{2}$. In fact, after the field $\phi_{1}$ settles down to $f$, it is more appropriate to speak about the phase $\theta$ of the original field $\phi$. We keep the notation $\phi_{2}$, with understanding that $\theta=\phi_{2} / f$.

Let us first estimate the amplitude of perturbations $\delta \phi_{2}$ at the time $\eta_{f}$ when the background field $\phi_{1}$ reaches $f$. At that time one has

$$
\chi_{1}\left(\eta_{f}\right) \sim \frac{1}{h\left(\eta_{*}-\eta_{f}\right)} \sim a_{f} \cdot f,
$$

where the subscript $f$ refers to the time $\eta_{f}$. From (7) we obtain

$$
\Delta_{\phi_{2}}\left(\eta_{f}\right)=\frac{\Delta_{\chi_{2}}\left(\eta_{f}\right)}{a_{f}} \simeq \frac{1}{2 \pi} h f .
$$

We now have to convert these entropy perturbations into adiabatic ones.

Let us recall that the global $O(2)$ symmetry is broken near $|\phi|=f$. We have in mind axion-like picture, in which the energy scale of this breaking is smaller than $f$. Importantly, the phase field $\phi_{2}$, as any (pseudo-)Goldstone field, minimally couples to gravity [18]. From this point on, the discussion is parallel to that in the curvaton model.

Generically, $\phi_{2}=0$ is not a minimum of the scalar potential, so the radial evolution ends up at a slope of the potential for the pseudo-Goldstone field $\phi_{2}$. Let us assume that at the time $\eta_{f}$, the Hubble parameter exceeds the mass parameter of this pseudo-Goldstone field. In this case the field $\phi_{2}$ stays close to zero for some time ${ }^{2}$, then rolls down to the nearest minimum of its potential, oscillates there and eventually the oscillations decay into usual

\footnotetext{
${ }^{2}$ This is true both for expanding Universe and for contracting Universe dominated by matter with stiff equation of state, $w>1$.
} 
particles. We assume that the decay happens at the hot Big Bang stage of the cosmological expansion, when the Universe is either radiation-dominated, or temporarily dominated by the oscillating field $\phi_{2}$ itself. Let $M$ denote the distance to the nearest minimum of the potential for the filed $\phi_{2}$, so that the number of minima of the scalar potential is $N \sim f / M$. Then at the beginning of oscillations and until their decay, the perturbations in the energy density of the pseudo-Goldstone field are

$$
\frac{\delta \rho_{\phi_{2}}}{\rho_{\phi_{2}}} \sim \frac{\delta \phi_{2}\left(\eta_{f}\right)}{M}
$$

This leads to adiabatic perturbations with flat spectrum and the amplitude

$$
\Delta \sim r \frac{\Delta_{\chi_{2}}\left(\eta_{f}\right)}{a_{f} M} \sim r \frac{h f}{2 \pi M}
$$

where $r$ is the ratio of the energy density of the field $\phi_{2}$ to the total energy density at the time the oscillations decay.

The pseudo-Goldstone picture with a single minimum of the potential for the phase would correspond to $M \sim f$. If we insist on the constraint (14), the amplitude (16) of the resulting adiabatic perturbations would be too small in that case (barring fine tuning). On the other hand, with $N \sim f / M \gg 1$ the required amplitude $\Delta \sim 10^{-5}$ can obtained without fine tuning of other parameters ${ }^{3}$. As we discuss in the end of Section 4, the constraint (13), and hence (14) may in fact be unnecessary, so the mechanism may work for $N=1$ as well.

\section{Generalizing to non-linear radial field perturbations}

Let us come back to the non-linearity issue. Since the perturbations (12) increase in time faster than the background field (2), the evolution in the radial direction may become nonlinear at some stage. Naively, one would think that the analysis of Section 2 goes through only if this does not happen, i.e., if the perturbations $\delta \phi_{1}$ remain small up to the time $\eta_{f}$. Such a requirement would push the parameters of the model to a very contrived range: the coupling contant $h$ would have to be extremely small, the field value $f$ would have to be super-Planckian, and the number of minima of the scalar potential, $N \sim f / M$, would have to be very large. This is discussed in Appendix. However, we argue that non-linearity of the radial field perturbations does not spoil the result (16).

\footnotetext{
${ }^{3}$ This discussion assumes that after the rolling stage, the value of the phase of the field $\phi$ is generic. Alternatively, one could make use of the anthropic argument for explaining why the field $\phi_{2}$ ends up close to the minimum of its potential, i.e., why $M \ll f$, so that the amplitude of the matter perturbations is large enough, cf. Refs. [19, 20]. With this line of reasoning, having large number of minima of the potential for the pseudo-Goldstone field is certainly unnecessary.
} 
The argument is as follows. Once the inequality (13) is satisfied, all interesting scales exit the "horizon" when the linearized theory is still valid. Let $\eta_{\text {lin }}$ be some moment of time when the linearized theory is valid, but all interesting scales are already super-"horizon". At that time, $\delta \phi_{2}$ may be considered as classical random field whose spatial gradients are negigible. According to (7) and (2) this field is small, $\delta \phi_{2} / \phi_{1} \sim h$, and it evolves in the same way as $\phi_{1}$,

$$
\delta \phi_{2}(\eta)=\frac{\delta \phi_{2}\left(\eta_{l i n}\right)}{\phi_{1}\left(\eta_{l i n}\right)} \phi_{1}(\eta)
$$

The field $\phi_{1}(\mathbf{x}, \eta)=\phi_{1}(\eta)+\delta \phi_{1}(\mathbf{x}, \eta)$ is also classical random field which is homogeneous inside the "horizon". At late times this field becomes inhomogeneous, but on super-horizon scales only. The point is that the evolution of the entire system proceeds according to the homogeneous classical field equation. Therefore, the symmetry argument presented before eq. (4) tells that $\delta \phi_{2}$ is still given by (17), but now with $\phi_{1}=\phi_{1}(\mathbf{x}, \eta)$. Note that $\delta \phi_{2}$ remains small. The field $\phi_{1}$ reaches the value $f$ in the end, and at that time

$$
\delta \phi_{2}=\frac{\delta \phi_{2}\left(\eta_{l i n}\right)}{\phi_{1}\left(\eta_{l i n}\right)} f
$$

This coincides with the result of the linearized theory.

Another way to phrase this argument is to write the rolling field $\phi_{1}$, with long-ranged perturbations included, as

$$
\phi_{1}(\mathbf{x}, \eta)=\frac{1}{h a(\eta)\left[\eta_{* e f f}(\mathbf{x})-\eta\right]} .
$$

Comparing this expression with (12) at the time when the linearized theory is still valid, we find that $\delta \eta_{*}(\mathbf{x})=\eta_{* e f f}(\mathbf{x}, \eta)-\eta_{*}$ is long ranged random classical field whose fluctuation is

$$
\Delta_{\delta \eta_{*}}=\frac{3 h}{2 \pi k}
$$

At late times, when $\delta \eta_{*} \gtrsim\left(\eta_{*}-\eta\right)$, the dependence of $\phi_{1}(\mathbf{x}, \eta)$ on $\delta \eta_{*}$ becomes non-linear, but the expression (18) remains valid. The point is, again, that the expression (17) with $\phi_{1}=\phi_{1}(\mathbf{x}, \eta)$ is a solution to the linearized field equation for $\delta \phi_{2}$ at all times. To see this explicitly, let us show that $\left|\nabla \delta \phi_{2}\right| \ll \delta \phi_{2}^{\prime}$, so that spatial variation of $\eta_{* \text { eff }}$ can be neglected in that field equation, and the argument before eq. (4) indeed applies. Using (17) we write

$$
\frac{\left|\nabla \delta \phi_{2}\right|}{\delta \phi_{2}^{\prime}}=\left|\nabla \delta \eta_{*}\right| .
$$

Now, $\nabla \delta \eta_{*}$ is random field with fluctuation

$$
\Delta_{\left(\nabla \delta \eta_{*}\right)}=k \Delta_{\delta \eta_{*}} \sim h
$$


so the ratio (19) is indeed small. Since the final state of the super-"horizon" perturbation $\delta \phi_{2}$ is independent of the parameter $\eta_{*}$, we see that non-linearity of the evolution of $\phi_{1}$, encoded in the non-linear dependence of $\phi_{1}$ on $\delta \eta_{*}$, is irrelevant for the spectrum of $\delta \phi_{2}$.

The latter way of presenting the argument suggests that the condition (13) is in fact unnecessary. The only requirement that remains is that $h \ll 1$. This ensures that the perturbations $\delta \phi_{1}$ become non-linear long after they exit the "horizon", and the spatial variation of $\eta_{* \text { eff }}$ is irrelevant in the field equation for $\delta \phi_{2}$. Hence, the parameters of our model are constrained very weakly; in particular, there may exist just one minimum of the potential for the pseudo-Goldstone field, i.e., $M \sim f$.

\section{Conclusions}

We conclude this paper with a few remarks. First, the mechanism for converting the perturbations of $\phi_{2}$ into adiabatic perturbations, suggested in Section 2, is most probably not unique. For example, one can imagine adding other fields which interact with the pseudoGoldstone field $\phi_{2}$ so that the energy density of these fields, after $\phi_{1}$ relaxes to $f$, is linear in $\delta \phi_{2}$. In that case the simple formula (16) would not be valid.

Second, the resulting spectrum needs not be exactly flat. One expects that for conformal symmetry slightly broken during the stage of rolling $\phi_{1}$, the spectrum becomes tilted. The tilt may be present also if the global symmetry $(U(1)$ in our example) is explicitly broken at that stage. How strong is the variation of the tilt with $k$, and whether there are other properties of the spectrum in these cases, remains to be understood.

Third, if the coupling constant $h$ is not very small, one would expect sizeable nonGaussianity of the perturbations. Also, there may be an interplay between the perturbations $\delta \phi_{1}$ and $\delta \phi_{2}$ which may result in peculiar properties of the adiabatic perturbations. It would be interesting to see whether or not the latter may help discriminate our mechanism from others (say, from inflationary ones).

Fourth, as in many other cases, the generation of scalar perturbations in our model is unrelated to the generation of tensor modes. Thus, there is no reason to expect sizeable gravitational wave background in our scenario.

Finally, we left aside the issue of the initial conditions for the field $\phi$. We simply assumed that this field is spatially homogeneous and starts rolling from near the top of its quartic potential. Whether these initial conditions may emerge naturally in the context of some cosmological scenario remains unclear.

We hope to address these and other issues in future.

The author is indebted to D. Gorbunov, A. Khmelnitsky, E. Nugaev, G. Rubtsov and I. Tkachev for helpful discussions. This work has been supported in part by Russian Foundation for Basic Research grant 08-02-00473. 


\section{Appendix}

In this Appendix we discuss what kind of constraints on the parameters one would obtain by insisting that the entire evoluton of both perturbations $\delta \phi_{1}$ and $\delta \phi_{2}$ occurs in the linear regime, so that the analysis of Section 2 is literally valid. The strongest constraint comes from the requirement that the perturbations of the radial field $\delta \phi_{1}$ are small by the end of the regime of rolling $\phi_{1}$,

$$
\delta \phi_{1}\left(\eta_{f}\right) \sim \Delta_{\chi_{1}}\left(\eta_{f}\right) / a_{f} \ll f .
$$

Let us pretend that this inequality should be valid for length scales up to the present hori-

zon size $k_{\text {min }}^{-1}$; perturbations of longer wavelengths correspond to unobservable shift of the parameter $\eta_{*}$ in the whole visible Universe.

Making use of (15) and (12) one finds that the condition (20) gives

$$
\frac{k_{\min }}{a_{f}} \gg h^{2} f
$$

Given the very small value of $k_{\text {min }}$ of the order of the present Hubble parameter, the latter inequality impies that the coupling constant $h$ is extremely small. Indeed, assuming that the Universe did not expand significantly before the beginning of the conventional hot Big Bang stage (no inflation) and ignoring weak dependence on the effective number of degrees of freedom $g_{*}$, one finds that $a_{f} \gtrsim T_{0} / T_{\max }$, where $T_{\max }$ and $T_{0}$ are the maximum temperature in the Universe and the present temperature, respectively. Hence, the constraint (21) reads

$$
h^{2} f \ll \frac{k_{\min }}{T_{0}} T_{\max } \sim 10^{-29} T_{\max }<10^{-29} M_{P l} .
$$

The constraint (21) implies also that

$$
f \gg M_{P l}
$$

This comes out from the estimate of the temperature $T_{\text {end }}$ at which the oscillations of the pseudo-Goldstone field $\phi_{2}$ are converted into usual particles. At this temperature the perturbations $\delta \phi_{2}$ are transformed into adiabatic ones, and one requires that $T_{\text {end }} \gtrsim 100 \mathrm{GeV}$ in order that the baryon asymmetry and dark matter may be thermally produced afterwards. To estimate $T_{\text {end }}$ in our model, we notice that the oscillations of $\phi_{2}$ begin at the time $t_{\text {osc }}$ when $H\left(t_{o s c}\right) \sim \mu$ where $\mu^{2}$ is the curvature of the potential for the pseudo-Golstone field. Again ignoring the dependence on $g_{*}$ we obtain the energy densities of hot matter and the pseudo-Goldstone field at that time,

$$
\rho_{\text {rad }}\left(t_{\text {osc }}\right) \sim T^{4}\left(t_{\text {osc }}\right) \sim \mu^{2} M_{P l}^{2}, \quad \rho_{\phi_{2}}\left(t_{o s c}\right) \sim \mu^{2} M^{2}
$$


The oscillations end up when $\rho_{\text {rad }} / \rho_{\phi_{2}}=r \leq 1$, so one finds

$$
\frac{T_{\text {end }}}{T_{\text {osc }}} \sim \frac{a\left(t_{\text {osc }}\right)}{a\left(t_{\text {end }}\right)}=\frac{1-r}{r} \frac{\rho_{\phi_{2}}\left(t_{\text {osc }}\right)}{\rho_{\text {rad }}\left(t_{\text {osc }}\right)} .
$$

Hence

$$
T_{\text {end }} \sim \frac{(1-r) M^{2}}{r M_{P l}^{2}} \sqrt{\mu M_{P l}}
$$

Making use of (16) we find

$$
T_{\text {end }} \sim \frac{h^{2} f^{2} r(1-r)}{\Delta^{2} M_{P l}^{2}} \sqrt{\mu M_{P l}} .
$$

Finally, using (22) and inserting $\Delta \sim 10^{-5}$ we obtain

$$
T_{\text {end }} \ll\left(\frac{f \mu^{1 / 2}}{M_{P l}^{3 / 2}}\right) \mathrm{GeV} .
$$

Since $\mu^{2}$ is the curvature of the scalar potential, one has $\mu<M_{P l}$. Therefore, high enough value $T_{\text {end }} \gtrsim 100 \mathrm{GeV}$ can be obtained only for super-Planckian values of $f$. Together with (22) this implies that the coupling constant is extremely small. In turn, the smallness of $h$ and eq. (16) mean that the correct amplitude of perturbations can be obtained only for huge number of minima of the scalar potential, $N=M / F$.

\section{References}

[1] V. F. Mukhanov and G. V. Chibisov, JETP Lett. 33 (1981) 532 [Pisma Zh. Eksp. Teor. Fiz. 33 (1981) 549];

S. W. Hawking, Phys. Lett. B 115 (1982) 295;

A. A. Starobinsky, Phys. Lett. B 117 (1982) 175;

A. H. Guth and S. Y. Pi, Phys. Rev. Lett. 49 (1982) 1110;

J. M. Bardeen, P. J. Steinhardt and M. S. Turner, Phys. Rev. D 28 (1983) 679.

[2] G. Veneziano, Phys. Lett. B 265 (1991) 287;

M. Gasperini and G. Veneziano, Mod. Phys. Lett. A 8 (1993) 3701 [arXiv:hepth/9309023];

M. Gasperini and G. Veneziano, Astropart. Phys. 1 (1993) 317 [arXiv:hep-th/9211021].

[3] M. Gasperini and G. Veneziano, Phys. Rept. 373 (2003) 1 [arXiv:hep-th/0207130].

[4] J. Khoury, B. A. Ovrut, P. J. Steinhardt and N. Turok, Phys. Rev. D 64 (2001) 123522 [arXiv:hep-th/0103239];

J. Khoury, B. A. Ovrut, N. Seiberg, P. J. Steinhardt and N. Turok, Phys. Rev. D 65 (2002) 086007 [arXiv:hep-th/0108187]. 
[5] P. J. Steinhardt and N. Turok, Phys. Rev. D 65 (2002) 126003 [arXiv:hep-th/0111098]; J. Khoury, P. J. Steinhardt and N. Turok, Phys. Rev. Lett. 92 (2004) 031302 [arXiv:hepth/0307132];

P. J. Steinhardt and N. Turok, New Astron. Rev. 49 (2005) 43 [arXiv:astro-ph/0404480].

[6] J. L. Lehners, Phys. Rept. 465 (2008) 223 [arXiv:0806.1245 [astro-ph]].

[7] P. Creminelli, M. A. Luty, A. Nicolis and L. Senatore, JHEP 0612 (2006) 080 [arXiv:hepth/0606090].

[8] F. Finelli, Phys. Lett. B 545 (2002) 1 [arXiv:hep-th/0206112].

[9] J. L. Lehners, P. McFadden, N. Turok and P. J. Steinhardt, Phys. Rev. D 76 (2007) 103501 [arXiv:hep-th/0702153].

[10] E. I. Buchbinder, J. Khoury and B. A. Ovrut, Phys. Rev. D 76 (2007) 123503 [arXiv:hepth/0702154].

[11] P. Creminelli and L. Senatore, JCAP 0711 (2007) 010 [arXiv:hep-th/0702165].

[12] A. Notari and A. Riotto, Nucl. Phys. B 644 (2002) 371 [arXiv:hep-th/0205019];

F. Di Marco, F. Finelli and R. Brandenberger, Phys. Rev. D 67 (2003) 063512 [arXiv:astro-ph/0211276].

[13] J. K. Erickson, D. H. Wesley, P. J. Steinhardt and N. Turok, Phys. Rev. D 69 (2004) 063514 [arXiv:hep-th/0312009];

D. Garfinkle, W. C. Lim, F. Pretorius and P. J. Steinhardt, Phys. Rev. D 78 (2008) 083537 [arXiv:0808.0542 [hep-th]].

[14] K. Koyama and D. Wands, JCAP 0704 (2007) 008 [arXiv:hep-th/0703040].

[15] A. J. Tolley and D. H. Wesley, JCAP 0705 (2007) 006 [arXiv:hep-th/0703101].

[16] J. L. Lehners and P. J. Steinhardt, "Dark Energy and the Return of the Phoenix Universe," arXiv:0812.3388 [hep-th].

[17] P. Sikivie, Lect. Notes Phys. 741 (2008) 19 [arXiv:astro-ph/0610440].

[18] M. B. Voloshin and A. D. Dolgov, Sov. J. Nucl. Phys. 35 (1982) 120 [Yad. Fiz. 35 (1982) 213].

[19] M. Tegmark and M. J. Rees, Astrophys. J. 499 (1998) 526 [arXiv:astro-ph/9709058]. 
[20] A. D. Linde, Phys. Lett. B 201 (1988) 437;

F. Wilczek, "A model of anthropic reasoning, addressing the dark to ordinary matter coincidence," arXiv:hep-ph/0408167. 